\title{
Addressing men's health policy concerns in Australia: what can be done?
}

\author{
James A Smith ${ }^{1,2}$
}

Address: ${ }^{1}$ Department of Paramedic \& Social Health Sciences, Flinders University, Adelaide, South Australia, Australia and ${ }^{2}$ Discipline of Public Health, University of Adelaide, Adelaide, South Australia Australia

Email: James A Smith - james.smith@adelaide.edu.au

Published: 10 October 2007

Australia and New Zealand Health Policy 2007, 4:20 doi:10.1 186/1743-8462-4-20
Received: 21 May 2007

Accepted: 10 October 2007

This article is available from: http://www.anzhealthpolicy.com/content/4/I/20

(c) 2007 Smith; licensee BioMed Central Ltd.

This is an Open Access article distributed under the terms of the Creative Commons Attribution License (http://creativecommons.org/licenses/by/2.0), which permits unrestricted use, distribution, and reproduction in any medium, provided the original work is properly cited.

\begin{abstract}
There is a lack of consensus about what men's health constitutes in Australia. The absence of a widely accepted definition has been problematic for establishing state and national men's health policies. I consider that one impediment to the implementation of state and federal men's health policies has been a lack of willingness to approach men's health from a broad public health perspective. In particular, scant attention has been paid to exploring lay perspectives of how men define and understand health, and in turn, how these relate to significant policy problems such as men's health service use. I conclude by suggesting that a focus on men's lay perspectives of their health emerging from the United Kingdom and the Republic of Ireland provides a useful framework to guide men's health policy discussion in Australia.
\end{abstract}

\section{Background \\ Defining men's health as a policy problem}

In Australia professional interest in men's health has grown markedly over the past decade [1]. The last two years alone has seen both the Australian Medical Association and the Royal Australian College of General Practice release position statements relating to men's health $[2,3]$. The Medical Journal of Australia even dedicated a special edition to men's health in October 2006. While there are inconsistent ways of defining men's health, a common concern raised in almost all recent scholarship relating to men's health in Australia is the lack of commitment to developing and implementing men's health policies at state and federal levels [1-6]. This concern is best summarised by Greg Malcher the National Convenor of GPs4Men who claims:

Australia still has no national men's health policy, despite the existence of a women's health policy since 1989. It would be naïve to suggest that simply developing a policy would be sufficient to deal with all the challenges of men's health - policy without adequately funded programs = "piffle". Yet, for those of us involved in men's health, there remains an overwhelming desire to see a formal acknowledgement by the federal government (whether a policy, position statement or other document) of the broad and unique issues of men's health, and a preparedness to fund a national program to address these issues. [6]

Currently, New South Wales is the only state in Australia to have succeeded in producing a men's health policy document that has been endorsed by a state Health Minister $[5,7]$. It is worth noting, however, that this document Moving Forward in Men's Health - was never explicitly labelled as a policy. This is a clear indication of the reluctance to use the word policy in the context of men's health. So why is there a reticence to implement state and national men's health policies in Australia and what can be done to rectify this significant public health concern? 
Firstly, debates relating to men's health policy development in Australia are not new. Indeed, there has been ongoing policy discussion at state and federal levels for quite some time [8-10]. A review of relevant literature reveals that many draft men's health policy documents have been developed during the past two decades, but that there have been major impediments to their formal endorsement and subsequent implementation [10]. There are four broad issues relating to the preclusion of men's health policy from state and federal agendas in Australia. These relate to medical dominance, the lack of a men's social movement, the Australian political and policy climate, and aspects of Australian men's culture [10]. Other commentators have argued that the lack of a well articulated theoretical orientation to direct men's health policy development has been problematic [11]. Divergent, narrow or inadequate definitions of men's health have also hindered policy responses $[5,10]$. It is this latter concern that I discuss in this paper.

\section{Discussion}

\section{Defining men's health: A policy problem}

'Men's health' is a term frequently used by the media, academics, health practitioners and the general public. However, there are subtle differences between how men's health can, or should, be defined $[5,10,12,13]$. While there is a wide recognition that men's health extends beyond male-specific conditions of the reproductive organs, such as prostate problems, testicular concerns and erectile dysfunction, these concerns have remained a prominent feature of the international discourse relating to men's health [5]. Epidemiological data has also been a central feature of men's health commentary, with comparisons between the status of men's and women's health predominating this discussion [10]. These definitions have contributed to a broader conceptualisation which suggests that men's health is perceived as being akin to a disease or condition unique to men, more prevalent in men, more serious among men, for which risk factors are different for men or for which different interventions are required for men [14]. Yet, even this definition has its limitations.

More recent commentary has drawn attention to the usefulness of understanding men's health in relation to social and economic determinants of health $[1,5]$. As such, health equity has become a central focus of this contemporary men's health discourse, where an emphasis has been placed on shifting resources towards the most vulnerable and disadvantaged groups of men $[1,5]$. Other considerations which complicate efforts to define men's health have also emerged, such as the burgeoning body of research relating to hegemonic masculinity and multiple masculinities [1]. At this juncture it is worth considering what this definitional dilemma means for men's health policy development in Australia. Collectively these understandings of men's health raise concern over whether men's health policy discussion should be focused on mainstream men's health issues - where all men are perceived to be the same, vulnerable groups of men - where variation between men is acknowledged, or perhaps both?

Irrespective of the policy approach advocated, the above evidence clearly demonstrates that a broader, more appropriate view of men's health is needed to develop a comprehensive national policy $[4,5,15]$. One way of embedding a broader conceptualisation of men's health into the current policy discussion, and one which has remained almost entirely absent from this discussion to date, is the inclusion of lay perspectives of health. There is no academic scholarship originating in Australia, of which the author is aware, that specifically links men's lay perspectives of their health with key policy concerns relating to men's help seeking practices, health service use and the way in which men navigate the current health system

\section{Closing the gap: Lay knowledge in men's health policy discussion}

Public health commentators have argued that there is a need to move beyond traditional forms of scientific knowledge to guide development of both healthy public policy and local public health programs [16-18]. In particular, previous commentary has shown that lay perspectives are particularly useful in understanding and addressing significant public health concerns $[17,19-22]$. Moreover, qualitative studies exploring lay knowledge are considered to be more persuasive in influencing policy makers than expert knowledge [18]. When there are differences in perspective among stakeholders in how to address particular health issues, as is the case in men's health, there is a need to explore the interface between professional and community understandings to maximise potential health gains $[23,24]$. A criticism of the discourse employed by health professionals about men's health particularly that associated with hegemonic masculinity has been the perpetuation of a 'men behaving badly' stance $[5,25]$. The inclusion of lay perspectives of men's health increases the capacity to move beyond this maledeficit model by providing an opportunity to understand men as real people, who live, work and play within multiple communities [1,5,25-28].

To provide a more persuasive men's health policy argument in Australia, and to facilitate a broader conceptualisation of what men's health constitutes, male consumer viewpoints ought to be considered when describing men's health. Yet, specific empirical data on male lay perspectives of health and well-being have largely remained absent in research on men's health [22], and this has been a contributing factor that has stalled the development and 
implementation of men's health policy in Australia [10]. Indeed, successes in women's health policy development in Australia have arisen out of a political discourse that has paid particular attention to women's lived experiences [10]. While it would seem sensible to conduct such research with men, there has been limited stimulus to determine men's understandings of health and well-being in Australia [10]. Yet, this has not been the case in other parts of the world.

There is a growing body of public health research emerging from the Republic of Ireland, Scotland and England which has shown an appreciation of lay perspectives of men's health $[22,26,29]$. This has assisted in understanding how 'health' is conceptualised differently between marginalised groups of men, such as gay men and disabled men. More importantly this has been used to describe their differential use of health services $[22,27]$. For example, the way in which men interpret, and respond to their chest pain [26], or the way in which men conceptualise their health, particularly in relation to risk $[22,29]$. While there is little evidence of the effective translation of this research into policy discussion, potential exists to do so. Interestingly, gender sensitive care in Britain has been supported by a policy emphasis on the importance of eliminating inequalities in the provision of health care, which intersects with this type of exploratory research $[27,30]$. Likewise, men's health discussion papers considered to be precursors to the development of a men's health policy in the Republic of Ireland have also paid attention to the ways men define certain aspects of their health [29].

\section{Conclusion}

The aim of improving the health status of men should, undoubtedly, be focused on developing valid and reliable data on men's perceptions of their health, their health practices and their health needs [31]. More importantly this data must be used to advocate for, and frame, emerging men's health policy responses in Australia. Of course, there are other considerations such as financial constraints and shifting timescales that influence this approach [32]. However, it is time for Australian men's health researchers, practitioners and policy makers to consider the achievements of their colleagues in the UK and the Republic of Ireland to adopt a consumer-focused public health response to develop and implement a national men's health policy here in Australia. Political will is required to make this happen.

\section{Acknowledgements}

I would like to acknowledge the support of the Masonic Foundation Inc through the Trevor Prescott Freemasons Memorial Scholarship. I would also like to thank Dr Murray Drummond, Mr Peter Lumb and Ms Natasha Houston for providing useful feedback on earlier drafts of this paper. This paper is dedicated to a dear colleague, the late Gary Bowes, who was an inspirational and passionate men's health advocate.

\section{References}

I. Smith J: Beyond masculine stereotypes: Moving men's health promotion forward in Australia. Health Promotion Journal of Australia 2007, I 8(I):20-25.

2. Australian Medical Association (AMA): Australian Medical Association position statement of men's health. Released on 4th April 2005 . (accessed via http://www.ama.com.au/web.nsf/doc/WEEN-6B56Y2/\$file/ Mens Health.pdfon the 19th May 2007).

3. Royal Australian College of General Practice (RACGP): Men's health: The Royal Australian College of General Practitioners' position statement on the role of general practitioners in delivering health care to Australian men. Policy endorsed by the 48th RACGP Council 5th August 2006 . (accessed via http://www.racgp.org.au/Content/NavigationMenu/Advocacy/ RACGPpositionstatements/200609MensHealth.pdf on 19th May 2007).

4. Gregory A, Lowy M, Zwa N: Men's health and well-being: Taking up the challenge in Australia. Medical Journal of Australia 2006, I 85(8):4 I I.

5. Macdonald J: Shifting paradigms: A social determinants approach to solving problems in men's health policy and practice. Medical Journal of Australia 2006, I 85(8):456-458.

6. Malcher G: "What is it with men's health?" - Men, their health and the system: a personal perspective. Medical Journal of Australia 2006, I 85(8):459-460.

7. NSW Department of Health: Moving forward in men's health Gladesville; Better Health Centre; 1999.

8. Connell R, Schofield T, Walker L, Wood J, Butland D, Fisher J, Bowyer J: Men's health: A research agenda and background report Canberra: Commonwealth Department of Health \& Aged Care; 1998.

9. Schofield T, Connell RW, Walker L, Wood JF, Butland DL: Understanding men's health and illness: A gender-relations approach to policy, research and practice. J Am Coll Health 2000, 48(6):247-256.

10. Lumb P: Why is men's health and well-being policy not implemented in Australia? International Journal of Men's Health 2003, 2(I):73-88.

II. Edwards P: Men's health: social policy and the new man. Social Alternatives 1997, I 6(2):55-57.

12. Banks I: New models for providing men with health care. Journal of Men's Health \& Gender 2004, I(2/3): I55-I 58.

13. Courtenay W: Teaming up for the new men's health movement. The Journal of Men's Studies 2000, 8(3):387-392.

14. Fletcher R: The development of men's health in Australia. In Promoting men's health: A guide for practitioners Edited by: Davidson N, Llyod T. London: Balliere Tindall; 200I.

15. White $A$, Fawkner $H$, Holmes $M$ : Is there a case for differential treatment of young men and women? Medical Journal of Australia 2006, I 85(8):454-455.

16. Williams G, Popay J: Social science and the future of population health. In The challenge of promoting health Edited by: Jones L, Sidell M. London: Open University Press; 1997.

17. Popay J, Williams G: Theorising inequalities in health: The place of lay knowledge. Sociology of Health \& IIlness 1998, 20(5):6 I 9-644.

18. Bryant T: Role of knowledge in public health and health promotion policy change. Health Promotion International 2002, I 7(I):89-98.

19. Entwistle V, Renfrew M, Yearley S, Forrester J, Lamont T: Lay perspectives: Advantages for health research. British Medical Journal 1998, 3 I 6(7 I 29):463-466.

20. Hogg C, Williamson C: Whose interests do lay people represent? Towards an understanding of the role lay people as members of committees. Health Expectations 200I, 4(I):2-9.

21. Lawton J: Lay experiences of health and illness: past research and future agendas. Sociology of Health \& Illness 2003, 25(3):23-40.

22. Robertson S: 'Not living life in too much of an excess': Lay men understanding health and well-being. Health (London) 2006, I0(2): I75-189.

23. Ansari W, Phillips C, Zwi A: Narrowing the gap between academic professional wisdom and community lay knowledge: perceptions from partnerships. Public Health 2002, I | 6(3): $151-159$. 
24. Sullivan M: The new subjective medicine: taking the patient's point of view on health care and health. Soc Sci Med 2003, 56(7): 1595-1604.

25. Smith J, Braunack-Mayer A, Wittert G: What do we know about men's help-seeking and health service use? Medical Journal of Australia 2006, 184(2):8I-83.

26. White A, Johnson M: Men making sense of their chest pain niggles, doubts and denials. Journal of Clinical Nursing 2000, 9(4):534-54I.

27. Williams R, Robertson S: Masculinities, men and promoting health through primary care. Primary Health Care 2006, I 6(8):25-28.

28. McVittie C, Willock J: "You can't fight windmills": How older men do health, ill health, and masculinities. Qualitative Health Research 2006, 16(6):788-80I.

29. Richardson N: Getting inside men's health Kilkenny: Health Promotion Department, South Eastern Health Board; 2004.

30. Miers M: Developing an understanding of gender sensitive care: Exploring concepts and knowledge. Journal of Advanced Nursing 2002, 40(I):69-77.

31. Laws T: A handbook of men's health Edinburgh: Churchill Livingstone; 2006.

32. Elliott H, Popay J: How are policy makers using evidence? Models of research utilisation and local NHS policy making. J Epidemiol Community Health 2000, 54(6):46I-468.

Publish with Bio Med Central and every scientist can read your work free of charge

"BioMed Central will be the most significant development for disseminating the results of biomedical research in our lifetime. "

Sir Paul Nurse, Cancer Research UK

Your research papers will be:

- available free of charge to the entire biomedical community

- peer reviewed and published immediately upon acceptance

- cited in PubMed and archived on PubMed Central

- yours - you keep the copyright 